\title{
The effect of comorbidities on COPD assessment: a pilot study
}

\author{
This article was published in the following Dove Press journal: \\ International Journal of COPD \\ 25 February 2015 \\ Number of times this article has been viewed
}

\section{Ulla Møller Weinreich ${ }^{1-3}$ \\ Lars Pilegaard Thomsen ${ }^{2}$ \\ Barbara Bielaska ${ }^{4}$ \\ Vania Helbo Jensen ${ }^{5,6}$ \\ Morten Vuust ${ }^{4}$ \\ Stephen Edward Rees ${ }^{2}$}

'Department of Respiratory Diseases, Aalborg University Hospital, Aalborg,

Denmark; ${ }^{2}$ Respiratory and Critical

Care Group (RCARE), Centre for

Model-Based Medical Decision

Support Systems, Department

of Health Science and Technology,

Aalborg University, Aalborg,

Denmark; ${ }^{3}$ The Clinical Institute,

Aalborg University Hospital, Aalborg,

Denmark; ${ }^{4}$ Department of Radiology,

Vendsyssel Hospital, Hjørring,

Denmark; ${ }^{5}$ Department of Radiology,

Horsens Regional Hospital, Horsens,

Denmark; 'Department of Radiology,

Aalborg University Hospital, Aalborg,

Denmark
Correspondence: Ulla Møller Weinreich Department of Respiratory Diseases, Aalborg University Hospital,

Mølleparkvej 4, DK-9000 Aalborg,

Denmark

Tel +459766 4735

Fax +459766 3577

Email ulw@rn.dk
Introduction: Patients with chronic obstructive pulmonary disease (COPD) frequently suffer from comorbidities. COPD severity may be evaluated by the Global initiative for chronic Obstructive Lung Disease (GOLD) combined risk assessment score (GOLD score). Spirometry, body plethysmography, diffusing capacity of the lung for carbon monoxide (DLCO), and high-resolution computed tomography (HR-CT) measure lung function and elucidate pulmonary pathology. This study assesses associations between GOLD score and measurements of lung function in COPD patients with and without $(\leq 1)$ comorbidities. It evaluates whether the presence of comorbidities influences evaluation by GOLD score of COPD severity, and questions whether GOLD score describes morbidity rather than COPD severity.

Methods: In this prospective study, 106 patients with stable COPD were included. Patients treated for lung cancer were excluded. Demographics, oxygen saturation $\left(\mathrm{SpO}_{2}\right)$, modified Medical Research Council Dyspnea Scale, COPD exacerbations, and comorbidities were recorded. Body plethysmography and DLCO were measured, and HR-CT performed and evaluated for emphysema and airways disease. COPD severity was stratified by the GOLD score. Correlation analyses: 1) GOLD score, 2) emphysema grade, and 3) airways disease and lung function parameters, described by: forced expiratory volume in the first second in percent of expected value $\left(\mathrm{FEV}_{1} \%\right)$, inspiratory capacity (IC\%), total lung volume (TLC\%), IC/TLC, and $\mathrm{SpO}_{2}$. Correlation analyses between subgroups and hierarchical cluster analysis were performed.

Results: Significant associations were found between GOLD score and both emphysema grade (correlation coefficients [cc]: $-0.2, P=0.03$ ) and lung function parameters (cc: -0.5 to -0.7 , $P$-values all $<0.001$ ) weakened in patients with $>1$ comorbidity (cc: -0.4 to $-0.5, P$-values all 0.001). Significant differences between subgroups were found in GOLD score and both $\mathrm{FEV}_{1} \%$ (cc: $-0.2, P=0.02)$ and IC/TLC (cc: $-0.2, P=0.02$ ). Comorbidities were associated with GOLD score and composite measures in hierarchical cluster analysis.

Conclusion: The presence of comorbidities influences the relationship between GOLD score and lung function measurements. GOLD score may be more representative of morbidity than of COPD severity.

Keywords: GOLD, diffusing capacity of the lung for carbon monoxide, high resolution computerized tomography, mMRC, total lung capacity, inspiratory capacity

\section{Introduction}

Chronic obstructive pulmonary disease (COPD) is characterized by a progressive and irreversible decline in lung function. ${ }^{1} \mathrm{COPD}$ is a highly heterogeneous disease and patients with COPD often suffer from several comorbidities. Recent studies have found patients to suffer from 4-6 comorbidities on average. ${ }^{2,3}$ Comorbidities have been shown to be associated with mortality ${ }^{3,4}$ and quality of life, ${ }^{5}$ and to influence the outcome of the Modified Medical Research Council Dyspnea Scale (mMRC) score. ${ }^{6}$ 
In order to estimate disease severity, as well as to understand COPD patients' symptoms, different types of measures are used. These include both patient-reported outcomes, ${ }^{7}$ which are often associated with the well-being of the patients, and more objective measures such as imaging ${ }^{8}$ and physiological testing, ${ }^{8}$ which measure different aspects of the disease. Often evaluation is a combination of different assessments as is the case of standardized assessments, such as the Global initiative for chronic Obstructive Lung Disease (GOLD) combined risk assessment score (GOLD score). ${ }^{1}$ However, a coalescence of measurements decided by the physician is often used to augment understanding of disease presentation in the individual patient.

The GOLD score is used in daily clinical care to stratify disease severity in COPD patients. ${ }^{9}$ It classifies patients in four strata, A to D, and is a composite measure of: 1) spirometry, where forced expiratory volume in the first second in percent of expected value $\left(\mathrm{FEV}_{1} \%\right)$ describes lung function impairment; 2) patients' symptoms, described by different symptom scores, as for example, the mMRC score; ${ }^{10}$ and 3) the number of exacerbations in the preceding year. ${ }^{9}$ The GOLD score describes the longitudinal behavior of COPD as well as the risk of exacerbation, hospitalization, and death increasing from group A to D. ${ }^{11}$ Despite the GOLD score being predictive of adverse events in the individual patient, there is heterogeneity in the presentation of the patients in the subgroups. As such, patients in group B have higher mortality and lower quality of life than patients in group C. ${ }^{12-14}$ Clinicians may therefore need a number of other measurements to interpret the patients' symptoms. This includes measures that provide visualization of the patients' pathoanatomical changes. As such, high-resolution computed tomography (HR-CT) demonstrates emphysema, ${ }^{15}$ and has been argued to be superior to lung function tests, as emphysematic changes may be detected on images prior to changes in lung function. ${ }^{16-18}$ In addition, airway wall thickness, measured by HR-CT scans, may reveal airways disease. ${ }^{19,20}$ Moreover, these HR-CT measurements are useful in understanding symptoms, as dyspnea has been shown to be associated with emphysema grade, and complaints of cough and phlegm have been associated with airways disease. ${ }^{20}$

The understanding of the COPD patient's symptoms may also be augmented by information obtained from other lung function measurements. Body plethysmography provides measurement of absolute lung volumes, which may help the clinician in understanding patients' respiratory complaints. Inspiratory capacity (IC) and inspiratory capacity-to-total lung capacity ratio (IC/TLC) have been shown to be useful measures of lung function, and to be strongly associated with dyspnea. ${ }^{21,22}$ In addition, the gas transport properties of the lungs, measured using the diffusing capacity of the lung for carbon monoxide (DLCO) has previously also been associated with dyspnea, ${ }^{23}$ and has been shown to be a strong predictor of longitudinal change in walking distance in COPD. ${ }^{24}$ As such, it may be descriptive of the physical condition of the COPD patient.

Taken together, a number of measures and patient-reported outcomes need to be taken into consideration, which makes the evaluation of the COPD patient very complex. Detailed measures of lung function and imaging may help us elucidate the pulmonary component of the disease, but the evaluation of COPD severity is complicated, as both COPD and comorbidities may influence the patients' well-being. An unanswered question is to what extent comorbidities influence the way COPD severity is evaluated by use of the GOLD score. If the GOLD score does not correlate well with pulmonary parameters, it could be argued that it is a measure of overall morbidity but does not reflect the pulmonary component of the disease. Thus, we hypothesized that in COPD patients with numerous comorbidities, disease severity is reflected by physiological and radiological findings but not by the GOLD score.

Hence, to evaluate the possible influence of comorbidities on the association between the GOLD score and lung function parameters as well as HR-CT findings, the specific aims of the present study are:

- To investigate the association between the GOLD score and lung function parameters such as DLCO, FEV, TLC, IC, IC/TLC, and oxygen saturation $\left(\mathrm{SpO}_{2}\right)$ in $\mathrm{COPD}$ patients with and without comorbidities;

- To investigate the association between the GOLD score and HR-CT findings.

Furthermore, as comorbidities are not expected to influence the outcome of objective measurements such as imaging and physiological measures:

- To investigate the association between HR-CT findings and lung function parameters such as DLCO, FEV TLC, IC, IC/TLC, and $\mathrm{SpO}_{2}$, in COPD patients with and without comorbidities.

\section{Materials and methods \\ Patients}

In this prospective study, a total of 111 patients suffering from COPD according to GOLD standards were included. ${ }^{9}$ Patients were recruited from the respiratory outpatient clinic at Aalborg University Hospital and the associated Rehabilitation 
Center and were all included by a pulmonary physician. All patients were in a stable state, with none having experienced COPD exacerbations within 6 weeks of inclusion and were treated for COPD in accordance to disease severity as advised by the GOLD recommendations. ${ }^{9}$ Furthermore, the Danish National hospital database Shared Medical Records were investigated to ensure that no major changes in treatment were made in either COPD or other chronic disease treatments 6 weeks prior to inclusion. Patients who had previously undergone lung surgery or who were suspected of lung cancer were excluded. Five patients were excluded from the final analysis as lung cancer was diagnosed as a coincidental finding to this study, leaving 106 patients in the total study population.

\section{Demographics and symptom scores}

At inclusion, demographic data on age, sex, and body mass index (BMI) were registered. Patients' smoking status was registered as never smokers, previous smokers, (when nonsmokers for 6 months or more), or current smokers, and the number of pack years was calculated. ${ }^{25}$ Furthermore, patients were asked to quantify symptoms according to the mMRC score $^{26}$ and to quantify how many exacerbations they had experienced during the preceding year. These data were used to stratify patients according to the GOLD score.

\section{Comorbidities}

The number of comorbidities was evaluated by a pulmonary physician. At inclusion, patients were asked to state their comorbidities. Furthermore, to ensure that no well-treated comorbidities were omitted, patients' prescriptions from Shared Medical Records were consulted. In addition, patients' case files were checked for ongoing contact with hospital departments other than the Department of Respiratory Diseases to reveal unnoticed, non-treated comorbidities. Comorbidities within the same International Classification of Diseases groups (ICD; v10) were not considered individually but counted as one disease entity.

\section{Lung function parameters}

A body plethysmography was performed on the day of the study and values computed with MasterScreen ${ }^{\circledR}$ PFT (VIASYS Healthcare GmbH, San Diego, CA, USA). The patient was introduced to the procedure by trained and experienced personnel and instructed throughout the process. The procedure was performed according to international standards, ${ }^{27}$ and international reference values were used. ${ }^{28}$ $\mathrm{FEV}_{1}, \mathrm{FEV}_{1} \%, \mathrm{FEV}_{1}$-to-forced vital capacity (FVC), TLC, and IC were recorded.
In continuation of the body plethysmography, DLCO measurement was performed according to international standards, ${ }^{29}$ using MasterScreen ${ }^{\circledR}$ Body (VIASYS Healthcare $\mathrm{GmbH}$ ). As for the plethysmography, the patient was also introduced to the procedure and instructed throughout the process by trained and experienced personnel. DLCO expressed in percent of the predicted value (DLCO\%) was used for analysis.

\section{HR-CT scans}

In 81 patients, a $0.625 \mathrm{~mm}$ sliced HR-CT scan and in 25 patients, a $1.25 \mathrm{~mm}$ sliced HR-CT scan were performed (Discovery CT750HD; General Electric Company, Fairfield, CT, USA) within 3 days of the lung function measurement. CT scans were anonymized and evaluated separately by three physicians, two radiologists, and one pulmonologist with special interest in thoracic imaging. The three physicians evaluated the HR-CT scans using the Picture Archiving and Communication System (PACS) solution (McKesson Radiology ${ }^{\mathrm{TM}}$, San Francisco, CA, USA), and as such allowed us to use the quantitative tools embedded in this system, combined with qualitative evaluation. The HR-CT scans were scored based on the CT evaluation performed by Aziz et $\mathrm{al}^{30}$ and the COPDGene group. ${ }^{19}$ Prior to evaluation, physicians were instructed in the scoring systems using a Powerpoint presentation. This presentation included visual reference material adapted from the COPDGene group, ${ }^{19}$ which was also available to the physicians during their evaluation of the HR-CT scans. Evaluations were carried out as follows:

- Three anatomical levels were identified; the upper level from the apex of the lung to the upper border of the aortic branch, middle section until $2 \mathrm{~cm}$ below the carina and lower level until $2 \mathrm{~cm}$ above the highest diaphragmatic dome. Each of these sections was scored visually, grading the degree of the lung affected by emphysema as $1-5$.

- The mean emphysema grade for the entire lung was calculated for each patient based on the physicians' evaluations: the mean value of the three physicians was computed for each patient and presented as the final emphysema grade in each case. A final score $\geq 2$ was considered emphysema, in accordance with Aziz et al. ${ }^{30}$

- The presence of airway disease was evaluated in the HR-CT scans; presence graded as 1 , absence as 0 . When at least two of the physicians graded the scans as 1 , the patient was evaluated as having airway disease.

In addition, concomitant radiological findings such as bronchiectasis were described according to radiological guidelines. ${ }^{31}$ 


\section{Statistics}

Statistical analysis on demographic data, correlation analyses, and Kappa values were done with SPSS software (IBM Corporation, Armonk, NY, USA). Correlation analyses on pairs of variables and hierarchical clustering were done in STATA (v13.1; StataCorp LP, College Station, TX, USA). $P$-values $<0.05$ were considered statistically significant.

Background data and comorbidities were presented as median and interquartile range (non-normally distributed). Based on the presence of comorbidities, the total study population was divided into two subgroups with 0-1 comorbidity and two or more comorbidities, respectively. In the total study population and both subgroups, the following analyses were made:

- To investigate the association between GOLD score and emphysema grade versus lung function parameters $\left(\mathrm{FEV}_{1} \%, \mathrm{DLCO} \%, \mathrm{IC} \%, \mathrm{TLC} \%, \mathrm{IC} / \mathrm{TLC}, \mathrm{SpO}_{2}\right)$, Spearman's correlation analyses were performed.

- To investigate the association between emphysema grade and the above mentioned lung function parameters, Pearson's correlation analyses were performed. Finally, Spearman's correlation analyses were used to investigate the association between the presence of airways disease and GOLD score as well as lung function parameters.

- Cohen's Kappa values ( $\kappa)$ were calculated to evaluate interobserver agreement on the presence of emphysema and airways disease.

- Equality of the correlation coefficients (cc) in the two groups was tested using the 'cortesti' command in STATA. ${ }^{32}$

- Hierarchical clustering is an exploratory technique used to discover groups of variables in the data. As such, it is not equipped with a $P$-value and can be seen as a hypothesisgenerating method. ${ }^{33}$
Hierarchical cluster analysis was calculated using the 'cluster' command in STATA, with single linkage and Euclidean distance. The variables included in the hierarchical cluster analysis $\left(\mathrm{L}^{2}\right)$ were: $\mathrm{DLCO} \%, \mathrm{IC} \%, \mathrm{IC} / \mathrm{TLC}, \mathrm{SpO}_{2}, \mathrm{mMRC}$, number of exacerbations, number of comorbidities, GOLD score, presence of airways disease, presence of emphysema, emphysema grade, and presence of emphysema and airways disease. The latter variable takes the values: 0 for patients with neither airways disease nor emphysema, 1 for patients with only one of them, and 2 for patients presenting both. All variables were standardized before the hierarchical clustering analysis.

\section{Ethical approval}

The study was approved by the local ethical committee of Northern Jutland, Denmark (N-20090012 and N-20140019) and all patients were recruited according to the Declaration of Helsinki; they were informed both orally and in writing, and all gave written consent to participation.

\section{Results \\ Demographics}

Background information on sex, age, BMI, postbronchodilator $\mathrm{FEV}_{1} \%$, FVC in percent of expected value (FVC\%), FEV $/$ FVC, mMRC score, smoking status, and number of pack years of the study population is presented in Table 1. The range of $\mathrm{FEV}_{1} / \mathrm{FVC}$ was $27-69$ and there was no significant difference in pre- and post-bronchodilator $\mathrm{FEV}_{1}, \mathrm{FVC}$, and $\mathrm{FEV}_{1} / \mathrm{FVC}$ (all $P$-values $>0.7$ ). Patients were classified according to the GOLD score as illustrated in Figure 1. Although patients with two or more comorbidities tended to be older, to have higher BMI and more pack years, there were no significant differences between any values when comparing subgroups with $0-1$ comorbidity and two or more comorbidities (all $P$-values $>0.09$ ).

Table I Background information on the total study population and two subgroups of patients; patients with 0-I comorbidity and patients with two or more comorbidities

\begin{tabular}{llll}
\hline & $\begin{array}{l}\text { Total study } \\
\text { population }(\mathbf{n}=\mathbf{l 0 6})\end{array}$ & $\begin{array}{l}\text { Patients with 0-I } \\
\text { comorbidity (n=50) }\end{array}$ & $\begin{array}{l}\text { Patients with two or } \\
\text { more comorbidities (n=56) }\end{array}$ \\
\hline Sex, female (\%) & 49 & 48 & 50 \\
Age, years, (median, quartiles) & $67(62-73)$ & $65(60-72)$ & $68(64-73)$ \\
BMI (median, quartiles) & $25(22-30)$ & $24(22-27)$ & $28(23-31)$ \\
FEV \% (median, quartiles) & $61(45-73)$ & $59(40-71)$ & $63(48-75)$ \\
FVC\% (median, quartiles) & $57(50-62)$ & $58(50-62)$ & $57(51-57)$ \\
FEV/FVC (median, quartiles) & $54(43-63)$ & $49(39-60)$ & $57(51-64)$ \\
mMRC (median, quartiles) & $2(2-3)$ & $1(1-2)$ & $2(I-2)$ \\
Smoking status: present/previous/never absolute numbers & $35 / 68 / 3$ & $15 / 34 / 1$ & $20 / 34 / 2$ \\
Pack years (median, quartiles) & $35(20-49)$ & $30(20-50)$ & $37(20-46)$ \\
\hline
\end{tabular}

Notes: Sex, age, BMI, FEV \%, FVC\%, FEV/FVC, mMRC score, smoking status, and number of pack years.

Abbreviations: BMI, body mass index; FEV \%, forced expiratory volume in the first second; FVC\%, forced vital capacity; mMRC, modified Medical Research Council. 


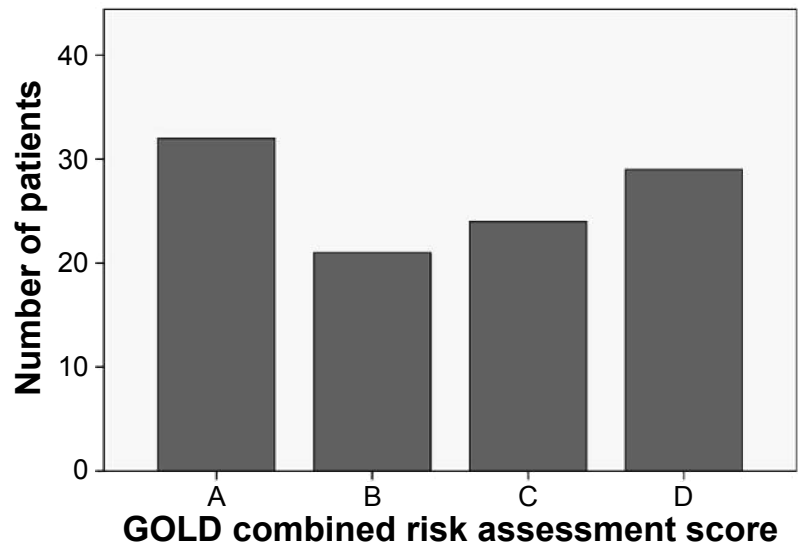

Figure I The study population distributed according to the GOLD combined risk assessment score of the individual patient.

Abbreviation: GOLD, Global initiative for chronic Obstructive Lung Disease.

\section{Comorbidities}

Patients had a median of three comorbidities (range 2-4). Figure 2 demonstrates the variability in the number of comorbidities per patient in the study population. Figure 3 shows the frequency of comorbidities registered in the study population. Malignant diseases included rectal cancer, vesical cancer, prostatic cancer, and breast cancer, with all of these in remission at the time of inclusion. Obesity was defined as BMI $>35$. Hematologic disorders were nonmalignant, and as such, did not require cytostatic treatment.

\section{GOLD score and lung function parameters}

Table 2 presents the association between the GOLD score and lung function parameters such as $\mathrm{FEV}_{1} \%, \mathrm{DLCO}^{2}$, $\mathrm{IC} \%, \mathrm{IC} / \mathrm{TLC}$, and $\mathrm{SpO}_{2}$ in the total study population and in subgroups.

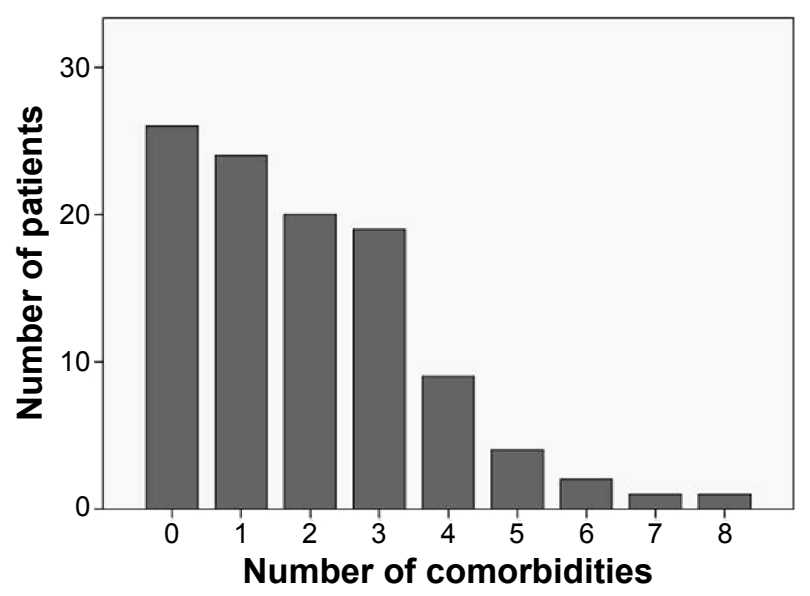

Figure 2 The study population $(\mathrm{N}=106)$ distributed according to the number of comorbidities in the individual patient.
In addition to the associations presented in Table 2, no association was found between TLC\% and GOLD score in the total study population or subgroups (all $P$-values $>0.7$ ).

\section{HR-CT findings}

Centrilobular emphysema was found in $77 \%(82 / 106)$ of the patients, paraseptal emphysema in 38\% (40/106) of the patients, and bullous emphysema in 34\% (36/106) of the patients. In 44\% (47/106) of the patients, more than one type of emphysema was seen. In the two subgroups, no significant differences in emphysema grade or in the number of patients with airways disease was seen (all $P$-values $>0.1$ ). Concomitant bronchiectasis was found in $9.4 \%(10 / 106)$ of the patients. The interobserver agreement of the presence of emphysema was $\kappa=0.9$ and the agreement on the presence of airways disease was $\kappa=0.4$ when evaluating the HR-CT scans.

\section{GOLD score and HR-CT findings}

A significant association was found between GOLD score and emphysema grade in the total study population (cc: -0.201 , $P=0.03$ ) and in patients with $0-1$ comorbidity (cc: -0.199 , $P=0.02$ ). No significant associations were found between the presence of GOLD score and airways disease.

\section{HR-CT findings and lung function parameters}

Table 3 shows the association between emphysema grade and lung function parameters in the total study population and in subgroups with $0-1$ comorbidity and two or more comorbidities.

In addition to the associations presented in Table 3, no association was found between emphysema grade and TLC $\%$ in either the total study population or in the two subgroups (all $P$-values $>0.8$ ).

Furthermore, associations between the presence of airways disease and GOLD score as well as lung function parameters were investigated. A significant association between the presence of airways disease and $\mathrm{FEV}_{1}(P=0.02)$ was found in the total study population.

\section{Combined correlation analysis and hierarchical cluster analyses}

The correlation between GOLD and $\mathrm{FEV}_{1}$ as well as between GOLD and IC/TLC were found to be significantly different between the subgroups with $0-1$ and two or more comorbidities (cc: -0.205 and $-0.212, P=0.02$ in both analyses).

Figure 4 shows the result of applying hierarchical cluster analysis to the dataset. Three clusters are apparent; reading 


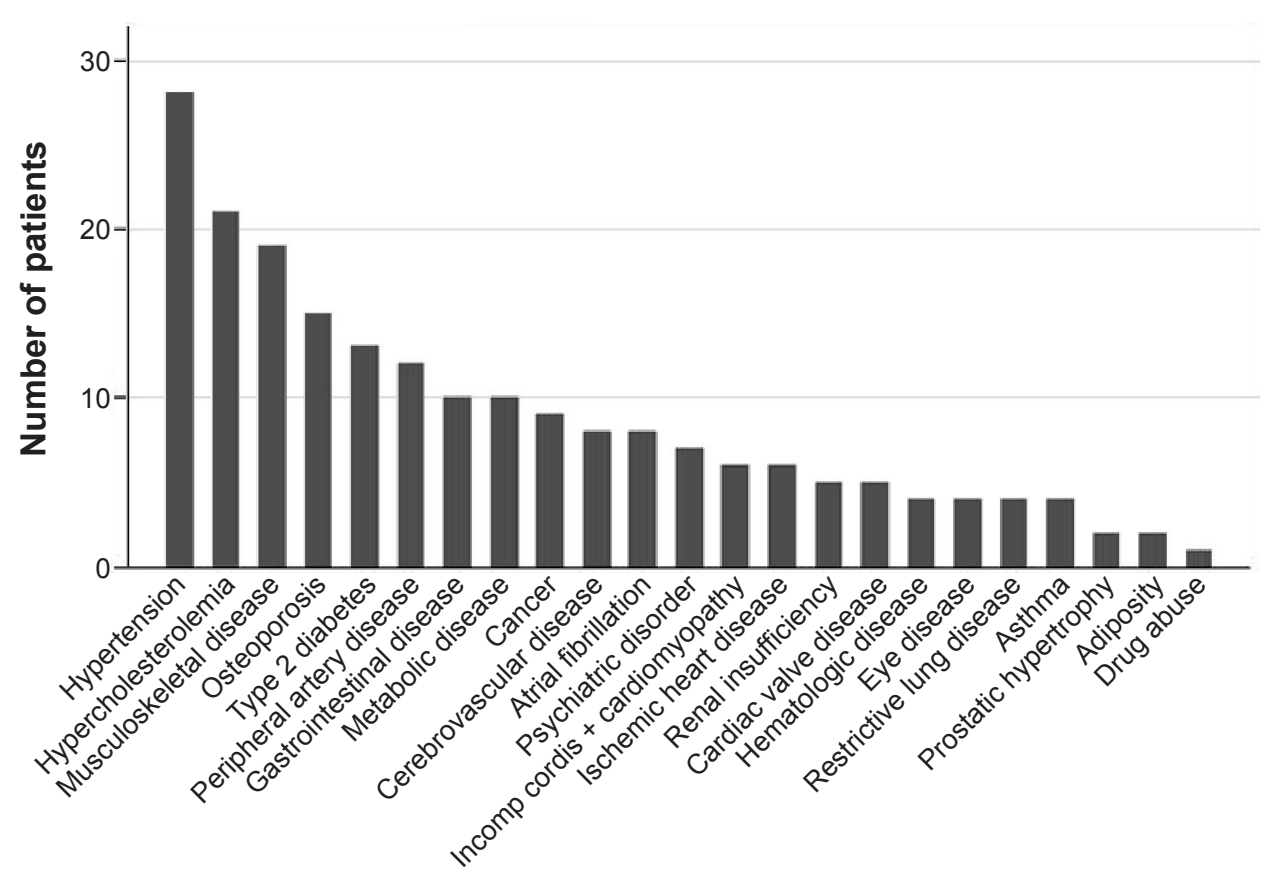

Figure 3 Comorbidities in absolute numbers in the study population $(\mathrm{N}=106)$.

left to right, these are: one with the lung function parameters, another one including the number of comorbidities, number of exacerbations, the GOLD score and the mMRC score, and a final cluster with the emphysema grade, and the presence of emphysema and/or airways disease. Comorbidities are the only parameter to cluster with GOLD score and composite patient-reported outcomes, ie, mMRC score and exacerbations.

\section{Discussion}

\section{Main findings}

This study investigates the influence of comorbidities on the associations between different evaluation tools in COPD.
In the total study population and in patients with $0-1$ comorbidity, significant associations were found between the GOLD score, lung function parameters, and emphysema grade. Although still significant, these associations were weaker in the subgroup with two or more comorbidities. Moreover, when correlating pairs of variables in the two subgroups, significantly different correlations between GOLD score and $\mathrm{FEV}_{1}$, as well as between GOLD score and IC/TLC were seen. Finally, when looking in the hierarchical cluster analysis, the number of comorbidities was the only variable that was closely associated with the GOLD score and two of the composite measures of the score, mMRC score, and exacerbations.

Table 2 Outcome, in terms of correlation coefficients and associated P-values, Spearman's correlation analyses of GOLD score versus FEV , in percent of expected value, DLCO in percent of expected value, IC in percent of expected value, the ratio between IC and TLC, and $\mathrm{SpO}_{2}$

\begin{tabular}{|c|c|c|c|c|c|c|}
\hline & \multicolumn{2}{|l|}{$\begin{array}{l}\text { GOLD score, total study } \\
\text { population }(n=106)\end{array}$} & \multicolumn{2}{|c|}{$\begin{array}{l}\text { GOLD score, patients with } 0-1 \\
\text { comorbidity }(n=50)\end{array}$} & \multicolumn{2}{|c|}{$\begin{array}{l}\text { GOLD score, patients with } \\
\text { two or more comorbidities }(n=56)\end{array}$} \\
\hline & Correlation coefficient & $P$-value & Correlation coefficient & $P$-value & Correlation coefficient & $P$-value \\
\hline $\mathrm{FEV}_{1} \%$ & -0.677 & $<0.001$ & -0.805 & $<0.001$ & -0.543 & $<0.001$ \\
\hline DLCO\% & -0.479 & $<0.001$ & -0.475 & $<0.001$ & -0.496 & $<0.00$ I \\
\hline IC\% & -0.584 & $<0.001$ & -0.670 & $<0.001$ & -0.504 & $<0.00$ I \\
\hline IC/TLC & -0.554 & $<0.001$ & -0.696 & $<0.00$ I & -0.426 & 0.001 \\
\hline $\mathrm{SpO}_{2}$ & -0.327 & 0.001 & -0.387 & 0.006 & -0.315 & 0.02 \\
\hline
\end{tabular}

Abbreviations: DLCO, diffusing capacity of the lung for carbon monoxide; $\mathrm{FEV}_{1}$, forced expiratory volume in the first second; GOLD, Global initiative for chronic Obstructive Lung Disease; IC, inspiratory capacity; TLC, total lung volume; $\mathrm{SpO}_{2}$, oxygen saturation. 
Table 3 Pearson's correlation analyses of emphysema grade versus FEV, in percent of expected value, DLCO in percent of expected value, IC in percent of expected value, the ratio between IC and TLC, and $\mathrm{SpO}_{2}$ in the total study population and in subgroups

\begin{tabular}{|c|c|c|c|c|c|c|}
\hline & \multicolumn{2}{|c|}{$\begin{array}{l}\text { Emphysema grade: total study } \\
\text { population }(n=106)\end{array}$} & \multicolumn{2}{|c|}{$\begin{array}{l}\text { Emphysema grade: patients with } \\
0-1 \text { comorbidity }(n=50)\end{array}$} & \multicolumn{2}{|c|}{$\begin{array}{l}\text { Emphysema grade: patients with } \\
\text { two or more comorbidities ( } n=56 \text { ) }\end{array}$} \\
\hline & Correlation coefficient & $P$-value & Correlation coefficient & $P$-value & Correlation coefficient & $P$-value \\
\hline $\mathrm{FEV}, \%$ & -0.281 & 0.004 & -0.418 & 0.002 & -0.138 & 0.3 \\
\hline DLCO\% & -0.454 & $<0.001$ & -0.480 & $<0.001$ & -0.454 & $<0.001$ \\
\hline IC\% & -0.192 & 0.05 & -0.191 & 0.2 & -0.198 & 0.2 \\
\hline IC/TLC & -0.198 & 0.04 & -0.258 & 0.07 & -0.149 & 0.3 \\
\hline $\mathrm{SpO}_{2}$ & -0.331 & 0.001 & -0.255 & 0.07 & -0.398 & 0.002 \\
\hline
\end{tabular}

Abbreviations: DLCO, diffusing capacity of the lung for carbon monoxide; FEV, forced expiratory volume in the first second; GOLD, Global initiative for chronic Obstructive Lung Disease; IC, inspiratory capacity; TLC, total lung volume; $\mathrm{SpO}_{2}$, oxygen saturation.

\section{The influence of comorbidities in COPD patients}

It is well-known that patients with COPD very frequently suffer from comorbidities. A recent study from general practice found comorbidities to be present in $90 \%$ of COPD patients, with $4 \pm 2$ comorbidities per patient. ${ }^{2}$ Furthermore, a study from tertiary care centers found an average of $6 \pm 3$ comorbidities per patient, ${ }^{3}$ a result that is consistent with our findings. In the present study, a number of observations indicate that GOLD score is potentially influenced by the number of comorbidities as follows.

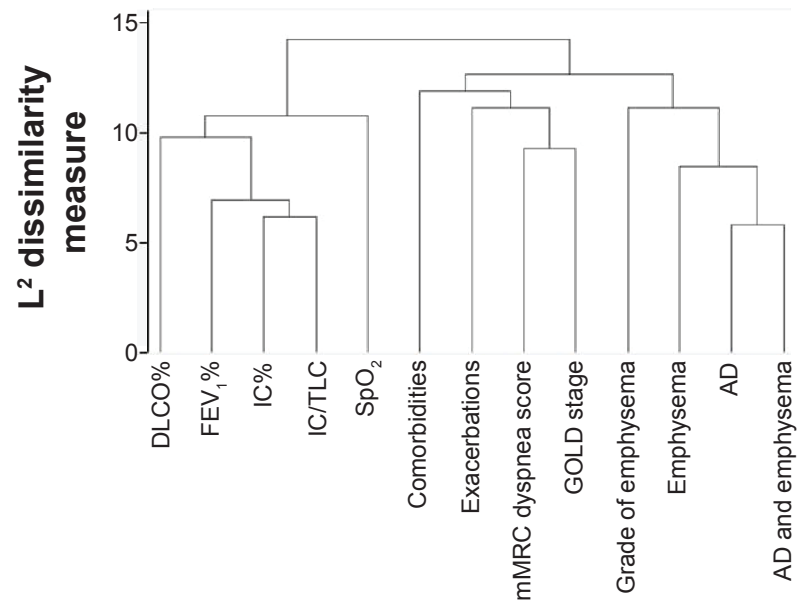

Figure 4 Hierarchical cluster analysis of lung function parameters, number of comorbidities, number of exacerbations, mMRC score, and GOLD score as well as evaluation of HR-CT scans for the presence of emphysema, AD or both emphysema and $A D$.

Notes: The figure shows: I) a cluster of parameters associated with lung function: $\mathrm{DLCO} \%, \mathrm{FEV}, \%, \mathrm{IC} \%$, and IC/TLC, and oxygen saturation $\left.\left(\mathrm{SpO}_{2}\right) ; 2\right)$ a cluster of comorbidities, number of exacerbations, mMRC score, and GOLD score; and 3) a cluster of emphysema grade, the presence of emphysema, the presence of $A D$, and the presence of either emphysema and AD.

Abbreviations: $A D$, airways disease; DLCO, diffusing capacity of the lung for carbon monoxide; FEV \%, forced expiratory volume in the first second; GOLD, Global initiative for chronic Obstructive Lung Disease; HR-CT, high-resolution computed tomography; IC, inspiratory capacity; mMRC, modified Medical Research Council; TLC, total lung capacity.
The data shown here indicate that the association between the GOLD score and measures of lung function parameters is weakened by the presence of comorbidities. A number of studies have demonstrated the influence of comorbidities on different evaluation methods used in COPD. For example, the study of Díez-Manglano et al demonstrated that patients with concomitant COPD and metabolic syndrome had significantly higher $\mathrm{FEV}_{1}$ and significantly more dyspnea than patients who suffered exclusively from COPD. ${ }^{34}$ In addition, the study of Güder et al showed that stratification of COPD by use of GOLD score is influenced by the presence of comorbidities. ${ }^{35}$ Moreover, it has been shown that the presence of comorbidities may influence the outcome of various symptom scores. ${ }^{6,36}$ In this sense, comorbidities may be effect modifiers when evaluating COPD. ${ }^{37}$

These findings are further supported by the fact that, when comparing correlations pairs of variables between the two subgroups, significant differences were found in the correlation between GOLD score and $\mathrm{FEV}_{1}$, as well as in the correlation between GOLD score and IC/TLC. As such, the association between GOLD score and lung function parameters was significantly weakened by the presence of comorbidities. Furthermore, when applying cluster analysis to the dataset, the number of comorbidities was the only parameter that was closely associated to the GOLD score and composite variables, the mMRC score, and the number of exacerbations. Interestingly, when using the cluster analysis, comorbidities and GOLD score were far more associated than GOLD score and $\mathrm{FEV}_{1}$, even though the latter is contained in the GOLD score.

In addition, a significant association between GOLD score and emphysema grade was seen in the total study population and in the subgroups with only $0-1$ comorbidity. However, no association was found between the GOLD 
score and emphysema grade in the group with two or more comorbidities. As COPD causes major morbidity as shown by many authors, ${ }^{38}$ such an association was not unexpected. In fact, it may support the hypothesis that the GOLD score is a measure of morbidity.

Taken together, these findings are indications that strongly support the hypothesis that the GOLD score may be a measure of overall morbidity rather than a measure of COPD severity.

Most of the associations in this study are consistent with the findings from previous studies. As such, a significant association between emphysema grade and both IC/TLC and DLCO was found in the total study population. This is consistent with previous studies, which have shown associations between emphysema and hyperinflation, as well as DLCO. ${ }^{17,39}$ Furthermore, a significant association between emphysema grade and $\mathrm{FEV}_{1}$ was seen in both the total study population and the 0-1 comorbidity subgroup, which is consistent with previous studies, although these have been of variable strength. ${ }^{39,40}$ Moreover, no association was found between TLC and emphysema grade, which is supported by previous findings. ${ }^{41}$ In addition, the significant associations found between the GOLD score and $\mathrm{FEV}_{1}, \mathrm{IC}$, and $\mathrm{SpO}_{2}$ in this study had also been reported previously. ${ }^{11,42}$ Taken together, the consistent findings between studies indicate that this study population can be generalized to represent other COPD populations.

One would expect that lung function parameters and HR-CT findings would be associated despite comorbidities. Surprisingly, the association between lung function parameters and emphysema grade was in fact weakened by the presence of comorbidities, apart from the association between emphysema grade and $\mathrm{SpO}_{2}$. However, a recent study from the COPD gene group is consistent with this finding. Bhatt et al reported that a subgroup of patients with mild COPD, characterized by older age, larger tobacco consumption, and presence of comorbidities, had more severe emphysematic changes than expected from their lung function. ${ }^{43}$ Further investigation in this subgroup is needed, as the reasons for these changes are not understood.

It is noticeable that the association between GOLD score and DLCO, as well as emphysema and DLCO does not seem to be influenced by the presence of comorbidities. This may be an incidental finding, but may also question our interpretation of DLCO. The latter is supported by the fact that the association between DLCO and GOLD is highly significant in both the total study population and in both subgroups. Thus, it may be speculated that in COPD patients, a decrease in DLCO may measure systemic deconditioning rather than gas exchange. However, this hypothesis will require further studies.

\section{Methodological considerations}

This study has a number of limitations. This is a pilot study, as reflected by the size of the study population, which may weaken the correlation analyses between subgroups. However, significant differences are seen when comparing pairs of variables, ie, the GOLD score versus FEV ${ }_{1}$ and GOLD score versus IC/TLC. This supports the hypothesis as does the hierarchical cluster analysis.

A large number of correlations are presented in this article, meaning that analysis is vulnerable to the presence of mass significance. To reduce the risk of this, comparisons between subgroups were only performed in parameters chosen a priori.

Lung function measurements require expertise and routine to be performed accurately. However, to limit the influence of interpersonal variation, all measurements were carried out by a small trained group of personnel.

HR-CT scans are increasingly being used to describe pathoanatomical changes in COPD. There are, however, a number of limitations in the evaluation of these scans, and there is debate as to which evaluation method is superior. In qualitative approaches such as visual scoring, interobserver variability in the interpretation exists. However, visual scoring has been described as a reliable method for interpreting, especially emphysema in CT scans. ${ }^{19,44}$ In this study, the interobserver agreement when evaluating emphysema is consistent with previous studies. ${ }^{45}$ In previous studies evaluating airways disease, interobserver agreement on the presence of airways disease has been seen as low as $0.3 .{ }^{45}$ As such, the interobserver agreement of this study is superior to this. This may be due to the fact that a validated reference material for visual scoring of CT scans was provided during the evaluation process. ${ }^{19}$ Furthermore, an evaluation method that relates radiological findings to other clinical outcomes was chosen. Taken together, the evaluation of the HR-CT scans has been carried out to try and challenge the obstacles of both interobserver variability and choice of method.

Finally, only a single scoring system was used to evaluate patients' symptoms. The results of this study may therefore not be representative if other symptom scores were used in stratification according to the GOLD score. However, other symptom scores such as the COPD assessment score, which may also be used as a composite measure 
in the GOLD score, have been proven to be similar to the mMRC score. ${ }^{46}$

In conclusion, this study indicates that the presence of comorbidities weaken the association between the GOLD score versus lung function parameters and emphysema grade. Furthermore, cluster analysis suggests an association between comorbidities and the GOLD score. These findings support the hypothesis that the GOLD combined risk stratification is a measure of overall morbidity, but does not necessarily reflect the pulmonary component of COPD. In future, comorbidities should therefore be accounted for when evaluating COPD. However, larger studies in this field are required.

\section{Acknowledgments}

We wish to thank statisticians Maria Rodrigo Domingo and Martin Bøgsted, Aalborg University Hospital, for their help with the statistical work in this article.

\section{Disclosure}

The authors report no conflicts of interest in this work.

\section{References}

1. Global Initiative for Chronic Obstructive Lung Disease. 2007 Update: Global Strategy for Diagnosis, Management, and Prevention of COPD. Available from: http://www.goldcopd.org/uploads/users/files/ GOLDReport07_0108.pdf. Accessed December 12, 2014.

2. García-Olmos L, Alberquilla A, Ayala V, et al. Comorbidity in patients with chronic obstructive pulmonary disease in family practice: a cross sectional study. BMC Fam Pract. 2013;14:11.

3. Divo M, Cote C, de Torres JP, et al; BODE Collaborative Group. Comorbidities and risk of mortality in patients with chronic obstructive pulmonary disease. Am J Respir Crit Care Med. 2012;186(2):155-161.

4. De Torres JP, Casanova C, Marín JM, et al. Prognostic evaluation of COPD patients: GOLD 2011 versus BODE and the COPD comorbidity index COTE. Thorax. 2014;69(9):799-804.

5. Koskela J, Kilpeläinen M, Kupiainen H, et al. Co-morbidities are the key nominators of the health related quality of life in mild and moderate COPD. BMC Pulm Med. 2014;14:102.

6. Barr RG, Celli BR, Mannino DM, et al. Comorbidities, patient knowledge, and disease management in a national sample of patients with COPD. Am J Med. 2009;122(4):348-355.

7. Jones $\mathrm{P}$, Miravitlles M, van der Molen T, Kulich K. Beyond FEV in COPD: a review of patient-reported outcomes and their measurement. Int J Chron Obstruct Pulmon Dis. 2012;7:697-709.

8. Fan L, Xia Y, Guan Y, Zhang TF, Liu SY. Characteristic features of pulmonary function test, $\mathrm{CT}$ volume analysis and MR perfusion imaging in COPD patients with different HRCT phenotypes. Clin Respir J. 2014; $8(1): 45-54$.

9. Global Initiative for Chonic Obstructive Lung Disease. Global Strategy for the Diagnosis, Management and Prevention of Chronic Obstructive Lung Disease: Updated 2013. Available from: http://www.goldcopd. org/uploads/users/files/GOLD_Report_2013_Feb20.pdf. Accessed December 12, 2014.

10. Mahler DA, Wells CK. Evaluation of clinical methods for rating dyspnea. Chest. 1988;93(3):580-586.

11. Agusti A, Edwards LD, Celli B, et al; ECLIPSE Investigators. Characteristics, stability and outcomes of the 2011 GOLD COPD groups in the ECLIPSE cohort. Eur Respir J. 2013;42(3):636-646.
12. Lange P, Marott JL, Vestbo J, et al. Prediction of the clinical course of chronic obstructive pulmonary disease, using the new GOLD classification: a study of the general population. Am J Respir Crit Care Med. 2012;186(10):975-981.

13. Soriano JB, Alfageme I, Almagro P, et al. Distribution and prognostic validity of the new Global Initiative for Chronic Obstructive Lung Disease grading classification. Chest. 2013;143(3):694-702.

14. Bolinde M, Tsiachristas A, Kruis A, Chavannes N, Rutten-van Mölken M. Relating the new COPD GOLD strategy to quality-of-life and costs in a primary care population. Eur Respir Soc. 2013;42(Suppl 57):P267.

15. Boulet LP, Turcotte H, Hudon C, Carrier G, Maltais F. Clinical, physiological and radiological features of asthma with incomplete reversibility of airflow obstruction compared with those of COPD. Can Respir J. 1998;5(4):270-277.

16. Choi $\mathrm{SH}$, Lee $\mathrm{CH}$. Yearly progression of low lung attenuation area in low dose CT scan for asymptomatic current smokers with normal lung function or GOLD stage 1 COPD. Am J Respir Crit Care Med. 2014;189(D43):A5954.

17. Smith BM, Austin JH, Newell JD Jr, et al. Pulmonary emphysema subtypes on computed tomography: the MESA COPD study. Am $J$ Med. 2014;127(1):94.e7-e23.

18. Newell JD Jr. CT of emphysema. Radiol Clin North Am. 2002;40(1): $31-42$, vii.

19. COPDGene CT Workshop Group; Barr RG, Berkowitz EA, Bigazzi F, et al. A combined pulmonary-radiology workshop for visual evaluation of COPD: study design, chest CT findings and concordance with quantitative evaluation. COPD. 2012;9(2):151-159.

20. Grydeland TB, Dirksen A, Coxson HO, et al. Quantitative computed tomography measures of emphysema and airway wall thickness are related to respiratory symptoms. Am J Respir Crit Care Med. 2010; 181(4):353-359.

21. Marin JM, Carrizo SJ, Gascon M, Sanchez A, Gallego B, Celli BR. Inspiratory capacity, dynamic hyperinflation, breathlessness, and exercise performance during the 6-minute-walk test in chronic obstructive pulmonary disease. Am J Respir Crit Care Med. 2001;163(6): 1395-1399.

22. Nishimura K, Yasui M, Nishimura T, Oga T. Airflow limitation or static hyperinflation: which is more closely related to dyspnea with activities of daily living in patients with COPD? Respir Res. 2011; 12:135.

23. Mahut B, Chevalier-Bidaud B, Plantier L, et al. Diffusing capacity for carbon monoxide is linked to ventilatory demand in patients with chronic obstructive pulmonary disease. COPD. 2012;9(1):16-21.

24. Farkhooy A, Janson C, Arnardóttir RH, Emtner M, Hedenström H, Malinovschi A. Impaired Carbon Monoxide Diffusing Capacity is the strongest lung function predictor of decline in 12 minute-walking distance in COPD; a 5-year follow-up study. COPD. Epub 2014 Sep 10.

25. Prignot J. Quantification and chemical markers of tobacco-exposure. Eur J Respir Dis. 1987;70(1):1-7.

26. Bestall JC, Paul EA, Garrod R, Garnham R, Jones PW, Wedzicha JA. Usefulness of the Medical Research Council (MRC) dyspnoea scale as a measure of disability in patients with chronic obstructive pulmonary disease. Thorax. 1999;54(7):581-586.

27. Wanger J, Clausen JL, Coates A, et al. Standardisation of the measurement of lung volumes. Eur Respir J. 2005;26(3):511-522.

28. Stocks J, Quanjer PH. Reference values for residual volume, functional residual capacity and total lung capacity. ATS Workshop on Lung Volume Measurements. Official Statement of The European Respiratory Society. Eur Respir J. 1995;8(3):492-506.

29. MacIntyre N, Crapo RO, Viegi G, et al. Standardisation of the single-breath determination of carbon monoxide uptake in the lung. Eur Respir J. 2005;26(4):720-735.

30. AzizZA, Wells AU, Desai SR, et al. Functional impairment in emphysema: contribution of airway abnormalities and distribution of parenchymal disease. AJR Am J Roentgenol. 2005;185(6):1509-1515.

31. Barker AF. Bronchiectasis. N Engl J Med. 2002;346(18):1383-1393. 
32. Cohen J, Cohen P. Applied Multiple Regression/Correlation Analysis for the Behavioral Sciences. 3rd ed. Mahwah, NJ: Lawrence Erlbaum Associates, Inc; 2003.

33. Stanford M. Chapter 7 Hierarchical cluster analysis. In: Statistics in Medicine. Vol 2. 3rd ed. London, UK: Elsevier, Inc; 2012:1-11.

34. Díez-Manglano J, Barquero-Romero J, Almagro P, et al; Working Group on COPD; Spanish Society of Internal Medicine. COPD patients with and without metabolic syndrome: clinical and functional differences. Intern Emerg Med. 2014;9(4):419-425.

35. Güder G, Brenner S, Angermann CE, et al. "GOLD or lower limit of normal definition? A comparison with expert-based diagnosis of chronic obstructive pulmonary disease in a prospective cohort-study". Respir Res. 2012;13(1):13.

36. Miyazaki M, Nakamura H, Chubachi S, et al; Keio COPD Comorbidity Research (K-CCR) Group. Analysis of comorbid factors that increase the COPD assessment test scores. Respir Res. 2014;15:13.

37. Bland M. An Introduction to Medical Statistics. New York, NY: Oxford University Press Inc.; 2000

38. Li LS, Caughey GE, Johnston KN. The association between comorbidities and physical performance in people with chronic obstructive pulmonary disease: a systematic review. Chron Respir Dis. 2014;11(1):3-13.

39. D’Anna SE, Asnaghi R, Caramori G, et al. High-resolution computed tomography quantitation of emphysema is correlated with selected lung function values in stable COPD. Respiration. 2012;83(5):383-390.
40. Boshetto P, Miniati M, Miotto D, et al. Predominant emphysema phenotype in chronic obstructive pulmonary disease patients. Eur Respir J. 2003;21(3):450-454.

41. Garfield JL, Marchetti N, Gaughan JP, Steiner RM, Criner GJ. Total lung capacity by plethysmography and high-resolution computed tomography in COPD. Int J Chron Obstruct Pulmon Dis. 2012;7:119-126.

42. Celli BR, Decramer M, Lystig T, Kesten S, Tashkin DP. Longitudinal inspiratory capacity changes in chronic obstructive pulmonary disease. Respir Res. 2012;13:66.

43. Bhatt SP, Sieren JC, Dransfield MT, et al; COPD Gene Investigators. Comparison of spirometric thresholds in diagnosing smoking-related airflow obstruction. Thorax. 2014;69(5):409-414.

44. Wille MM, Thomsen LH, Dirksen A, Petersen J, Pedersen JH, Shaker SB. Emphysema progression is visually detectable in low-dose CT in continuous but not in former smokers. Eur Radiol. 2014;24(11): 2692-2699.

45. Sundaram B, Gross BH, Martinez FJ, et al. Accuracy of high-resolution CT in the diagnosis of diffuse lung disease: effect of predominance and distribution of findings. AJR Am J Roentgenol. 2008;191(4): 1032-1039.

46. Jones PW, Adamek L, Nadeau G, Banik N. Comparisons of health status scores with MRC grades in COPD: implications for the GOLD 2011 classification. Eur Respir J. 2013;42(3):647-654.
International Journal of COPD

\section{Publish your work in this journal}

The International Journal of COPD is an international, peer-reviewed journal of therapeutics and pharmacology focusing on concise rapid reporting of clinical studies and reviews in COPD. Special focus is given to the pathophysiological processes underlying the disease, intervention programs, patient focused education, and self management protocols.

\section{Dovepress}

This journal is indexed on PubMed Central, MedLine and CAS. The manuscript management system is completely online and includes a very quick and fair peer-review system, which is all easy to use. Visit $\mathrm{http}: / /$ www.dovepress.com/testimonials.php to read real quotes from published authors. 